\title{
Life-time prediction for aviation GTE compressor disk based on mixed-mode and multi-axial fracture resistance parameters
}

\author{
Valery Shlyannikov ${ }^{1,1}$, Ivan Ishtyryakov $^{1}$, and Rustam Yarullin $^{1}$ \\ ${ }^{1}$ FRC Kazan Scientific Center of Russian Academy of Sciences, 420111 Kazan, Russia
}

\begin{abstract}
This study is concerned with analysis of fatigue crack growth and life-time prediction for aviation GTE compressor disk under operation conditions. For consideration were different combinations of rotational speed, temperature, surface flaw form and sizes as well as elastic-plastic titanium alloy BT3-1 properties are employed. A crack growth rate equation is derived involving the fracture process zone size and nonlinear stress intensity factor. The assessments of the structural integrity of the rotating disk are compared for elastic and elastic-plastic solutions. It is stated that the traditional elastic crack growth models overestimate the residual fatigue lifetime with respect to the nonlinear fracture mechanics approach.
\end{abstract}

\section{Introduction}

Today civil aviation GTE components design becomes very demanding due to high temperatures, complex mechanical loads, corrosive environment and long expected lifetimes. Such loading conditions lead to fatigue crack initiations and their propagation up to reaching a critical zone in rotating disks. Most of the critical zones are characterized by the presence of plastic deformations in which the effective stresses exceed the yield strength of the material at the corresponding temperature. These circumstances assume the application of nonlinear fracture and continuum damage mechanics approaches to aviation GTE components lifetime prediction. Shlyannikov et al. [1,2] performed extensive systematic theoretical and numerical studies of the nonlinear fracture mechanics problems for elastic-plastic power-law-hardening materials. The authors $[1,2]$ proposed new fracture resistance parameters for the elastic-plastic crack growth problems in the form of plastic stress intensity factors (SIF), which accounted for the undamaged and damaged crack tip stress-strain fields. It was found that the plastic fracture process zone sizes and $I_{n}$-factor were strong functions of the material constitutive equations, crack size, specimen configuration, and loading conditions, which were reproduced from nonlinear SIFs.

The present work provide an appropriate numerical study to part-through surface cracks lifetime predictions for rotating components of civil aviation gas-turbine engines. The full-size 3D FEM models of considered GTE compressor disk was developed with the aim to reproduce the in service damage accumulation and growth. The nonlinear stress intensity factors and fracture process zone sizes of a small corner inclined crack emanating from the slot key fillet in compressor disk are calculated using numerical procedures. For consideration were different combinations of rotational speed, temperature, surface flaw form and sizes as well as elastic-plastic titanium alloy BT3-1 properties are employed.

1

Corresponding author: shlyannikov@mail.ru 


\section{Low cycle fatigue and multiaxial material constitutive relations}

Based on the cyclic properties, in a similar manner to the monotonic Ramberg-Osgood equation, it is possible to use the equation for the cyclic stress-strain curve as the relationship between the stress range $\Delta \sigma$ and the plastic range $\Delta \varepsilon_{P}$ [3]:

$$
\frac{\Delta \sigma}{2}=K^{\prime}\left(\frac{\Delta \varepsilon_{P}}{2}\right)^{n^{\prime}},
$$

where $K^{\prime}$ is the cyclic strength coefficient and $n^{\prime}$ denotes the cyclic strain hardening exponent. The plastic strain energy per cycle is the area of the hysteresis loop, which is assumed to be representative at the half-life in the case of strain-controlled tests. Ellyin et al. $[3,4]$ studied the hysteresis absorption energy and its relationship with fatigue for both Masing and non-Masing materials. The relationship with the form [3]

$$
\Delta \sigma \Delta \varepsilon_{P}=4 \sigma_{f}^{*} \varepsilon_{f}^{*}\left(2 N_{f}\right)^{(b+c)},
$$

where $\Delta \varepsilon_{P}$ is the plastic strain range, $\varepsilon_{f}^{*}$ is the fatigue ductility coefficient, $b$ and $c$ is the fatigue ductility exponent is a function of the stress range and plastic strain range, and it has been used to describe the fatigue properties of a material. Considering the known approximation relationships of $K^{\prime}=1.65 \sigma_{u}$ and $\sigma_{f}^{*}=1.5 \sigma_{u}$ [5], where $\sigma_{u}$ is the ultimate true tensile strength, allows the number of reversals to failure $2 N_{f}$ to be determined by jointly solving Eqs. (1) and (2):

$$
N_{f}=2^{\phi}\left(0.275 \Delta \varepsilon_{P}^{1+n^{\prime}} / \varepsilon_{f}^{*}\right)^{1 / \gamma}, \text { where } \gamma=1 /(b+c) \text { and } \phi=\left(1-n^{\prime}-\gamma\right) / \gamma .
$$

From a structural integrity perspective, it is necessary to develop reliable methods for predicting the fatigue lifetime under multi-axial stress conditions. Many criteria have been proposed, and this indicates the complexity of the problem as well as the lack of an agreed unified approach. Shlyannikov [6] introduced the following equation for multi-axial limiting strain under low cycle fatigue using the general stress-based criterion:

$$
\Delta \varepsilon_{f}^{P}=\left[\frac{\Delta \varepsilon_{0}^{P}}{b_{i}}\left(\frac{\eta_{i}}{1-\chi+\chi \eta_{i}}\right)^{n^{\prime}}\right],
$$

where for the plane strain:

$$
b_{i}=\frac{2}{3} \sqrt{1-\xi+\xi^{2}} ; \xi=\frac{\lambda\left(1-v^{2}\right)-v(1+v)}{\left(1-v^{2}\right)-\lambda v(1+v)} ; \eta_{i}=\sqrt{1-\lambda+\lambda^{2}+v(v-1)(1+\lambda)^{2}} ;
$$

and $\Delta \varepsilon_{0}^{P}$ is the limiting cyclic plastic strain under uniaxial tension, $\chi$ is an experimental material constant determined as the ratio of the uniaxial tensile relative to the compression strength, i.e., $\chi=\sigma_{t} / \sigma_{c}, v$ is Poisson's ratio, and $\lambda=\sigma_{2} / \sigma_{1}$ is the principal stress ratio. In Eq. (4), the experimental material constant $\chi$ is the governing parameter in the PisarenkoLebedev criterion

$$
\sigma_{e q v}=\chi \sigma_{e}+(1-\chi) \sigma_{1}
$$

where $\sigma_{l}$ is the principal stress and $\sigma_{e}$ is the equivalent von Mises stress. For a ductile fracture, $\chi=1$, and for a brittle fracture, $\chi=0$. 


\section{Mixed mode crack growth parameters}

\subsection{Plastic stress intensity factors}

Shlyannikov and Tumanov [1] have extended the HRR-solution and introduced new numerical method to obtain an accurate description for the distribution along the crack front of the governing parameter for the elastic-plastic solution in the form of the plastic stress intensity factor (SIF). This method combining the knowledge of the dominant singular solution with the finite element technique. The plastic stress intensity factor $K_{P}$ in mixedmode small-scale yielding can be expressed directly in terms of the corresponding elastic stress intensity factors $K_{l}$ and $K_{2}$ using Rice's $J$-integral. That is

$$
\bar{K}_{P}=\left[\left(\frac{K_{e q v}}{\sigma_{y}}\right)^{2} \frac{(1+v)(1+\kappa)}{4 \alpha_{p} I_{n}^{f} L}\right]^{\frac{1}{n_{p}+1}}, K_{e q v}^{2}=\sqrt{\left(K_{1}^{2}+K_{2}^{2}\right)^{2}+4\left(K_{1} K_{2}\right)^{2}}
$$

is equivalent elastic stress intensity factor, $\kappa=3-4 v, v$ is Poisson's ratio, $\alpha_{p}$ is the strain hardening coefficient, $n_{p}$ is the strain hardening exponent, $\bar{r}=r / L$ is a dimensionless crack tip distance, $L$ is cracked body characteristic size, $\sigma_{y}$ is the yield stress and $I_{n}^{f}$ is the governing parameter of the crack-tip nonlinear stress-strain field. The numerical constant $I_{n}^{f}$ is obtained from the singularity analysis by means of the conjugation solutions for the far and near crack-tip fields.

The $J$ integral formulation for large-scale yielding conditions leads to the following the expression for the plastic SIF

$$
\bar{K}_{P}=\left(\frac{J_{f} E}{\alpha_{p} \sigma_{0}^{2} I_{n} L}\right)^{\frac{1}{n+1}}, K_{P}=\sigma_{0} w^{\frac{1}{n+1}} \bar{K}_{P}
$$

The $I_{n}$-factor in Eqs. $(7,9)$ is a numerical parameter related to the nonlinear crack tip field, which should be determined for the cracked body with a finite size. For full threedimensional (3D)-mixed mode problems, Shlyannikov and Tumanov [1] proposed the determination of the $I_{n}$-integral based on finite element analysis. Numerical results regarding the behavior of the $I_{n}$-integral in the most popular experimental configurations for test specimens in fracture mechanics as well as real structural elements were reported in previous studies $[1,2,7]$.

\subsection{Low-cycle fatigue crack growth rate model}

In order to derive the low cycle fatigue crack growth rate model, Ellyin suggested [3] modifying the crack-tip distance by considering the blunted crack tip radius $\rho$ after repeated loading and unloading cycles:

$$
r=\delta^{*}+\rho,
$$

where $\delta^{*}=\bar{r}_{f} a$. Therefore, the crack growth rate per cycle, $d a / d N$, is given as follows:

$$
\dot{a}_{f}=\frac{d a}{d N}=\frac{r_{f}}{\Delta N}
$$


At the threshold, $d a / d N \approx 0$ and the SIF reduces to $\Delta K_{\text {eqv }}=\Delta K_{t h}$; thus, from Eqs. (9) and (10), rearranging we obtain the low cycle crack growth rate equation as follows:

$$
\left(\frac{d a}{d N}\right)=2 \delta^{*}\left\{\left(\Delta K_{e q v}^{2}-\Delta K_{t h}^{2}\right)\left[\frac{(\kappa-1)(1+v)}{4 \pi}+\frac{n^{\prime}}{\left(n^{\prime}+1\right)} \frac{\tilde{\sigma}_{e}^{n^{\prime}+1}}{I_{n}}\left(\frac{a}{w}\right)\right] /\left(4 E \sigma_{f}^{*} \varepsilon_{f}^{*} \delta^{*}\right)\right\}^{\frac{1}{\beta}}
$$

where $\beta=-(b+c)$.

\section{Computational results}

\subsection{Material properties and loading conditions}

In the present study, we conducted a structural integrity assessment of the nonlinear fracture mechanics by considering an aviation gas turbine engine (GTE) compressor (Fig. 1 ,a) disk as a case study. A GTE disks are generally loaded with both cyclic and sustained centrifugal loads, as well as thermal loads because it is operated at a moderately high temperature. Part-through surface cracks occurred in the titanium disk and blade attachment of a compressor disk (Fig. 1,b). This "dovetail" type attachment had a broach angle of $26^{\circ}$ at the axis of rotation. Quarter-elliptical corner cracks also developed in slot fillets under the blades near the outer surface of the disk. Thus, we performed numerical investigations of the surface crack growth behavior for given combinations of the main parameters such as the rotational speed of the compressor, temperature, shape and size of the defect, and the elastic-plastic properties of the material. The combinations of parameters used in the numerical calculations are presented in Table 1.

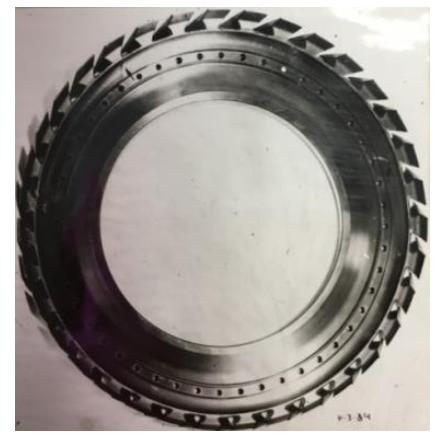

a)

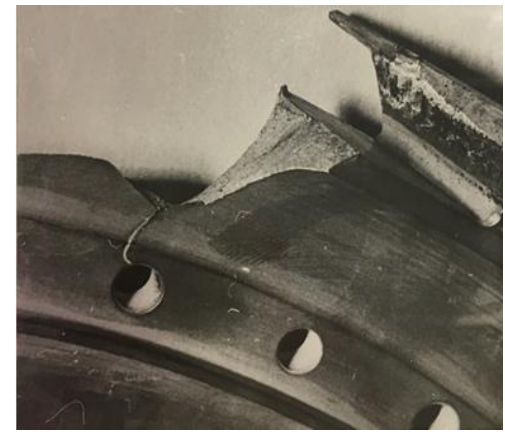

b)

Fig. 1. Compressor disk (a) and operation damage (b) to a disk-blade attachment by corner crack.

Table 1. Various parameters employed in the numerical calculations.

\begin{tabular}{|c|c|c|c|c|}
\hline \multirow{2}{*}{$\begin{array}{c}\text { Rotational speed } \\
(\mathbf{r a d} / \mathbf{s})\end{array}$} & $\begin{array}{c}\text { Temperature } \\
\left({ }^{\circ} \mathbf{C}\right)\end{array}$ & \multicolumn{3}{|c|}{$\begin{array}{c}\text { Surface crack sizes a/c } \\
(\mathbf{m m})\end{array}$} \\
\cline { 2 - 5 } & & 1st front & 2nd front & 3rd front \\
\hline \multirow{2}{*}{$925 / 1000$} & 23 & \multirow{2}{*}{$0.34 / 0.71$} & $0.76 / 1.32$ & $1.5 / 2.22$ \\
\cline { 2 - 2 } & 300 & & & \\
\cline { 2 - 3 } & 370 & & \\
\hline
\end{tabular}


Table 2. Static and cyclic properties of titanium alloy BT3-1.

\begin{tabular}{|l|c|c|c|}
\hline Material property & $\mathbf{+ 2 3}^{\circ} \mathbf{C}$ & $\mathbf{+ 3 0 0}^{\circ} \mathbf{C}$ & $\mathbf{+ 3 7 0}^{\circ} \mathbf{C}$ \\
\hline Yield stress $\boldsymbol{\sigma}_{\mathbf{0 . 2}},[\mathrm{MPa}]$ & 1004.49 & 753.26 & 699.1 \\
\hline Tensile stress $\boldsymbol{\sigma}_{\mathbf{t}},[\mathrm{MPa}]$ & 1073.04 & 889.0 & 856.72 \\
\hline Ultimate tensile strength $\boldsymbol{\sigma}_{\mathbf{u}},[\mathrm{MPa}]$ & 1602.0 & 1515.0 & 1500.43 \\
\hline Young's modulus $\mathbf{E},[\mathrm{MPa}]$ & 100098.6 & 96071.43 & 97553.8 \\
\hline Strain hardening exponent $\mathbf{n}$ & 12.88 & 8.915 & 8.43 \\
\hline Strain hardening coefficient $\boldsymbol{\alpha}$ & 1.11 & 0.9904734 & 0.974 \\
\hline Fatigue strength $\sigma_{f}^{*},[\mathrm{MPa}]$ & 2675 & 2530 & 2505 \\
\hline Fatigue ductility $\varepsilon_{f}^{*}$ & 0.35 & 0.35 & 0.35 \\
\hline Cyclic strain hardening exponent $\mathbf{n}^{\prime}$ & 9.09 & 9.09 & 9.09 \\
\hline Fatigue stress equation exponent $\mathbf{b}$ & -0.095 & -0.095 & -0.095 \\
\hline Fatigue plastic strain equation exponent $\mathbf{c}$ & -0.69 & -0.69 & -0.69 \\
\hline
\end{tabular}

The compressor disk considered for the numerical calculations comprised the twophase titanium alloy BT3-1. The main monotonic and low-cycle fatigue mechanical properties of the material analyzed at both room temperature and elevated temperatures are summarized in Table 2.

The structural integrity assessment for the GTE rotating disks addressed two distinct problems: numerical analyses of the stress-strain state under the operating loading conditions and crack growth modeling based on theoretical assumptions. Thus, full-field finite element analyses were performed using the ANSYS [8] FE code to determine the numerical parameters for the elastic-plastic crack-front fields in the form of the plastic strain range $\Delta \varepsilon_{P}, I_{n}$-factor, and dimensionless equivalent stresses $\left(\tilde{\sigma}_{e}^{n^{\prime}}\right)$. The numerical calculations were programmed according to Table 1 to analyze specific combinations of the rotational speed, surface flaw sizes, and main mechanical properties at different temperatures.

\subsection{Stress-strain fields}

The global finite element model represented one-quarter of a compressor disk with five blades (Fig.2a). Figure 2,b shows plots of the local elastic-plastic von Mises stress distributions and clearly indicates that the equivalent stress peak mainly occurred close to the free surface of the slot key where a corner crack was likely to nucleate and start to grow due to the cyclic loading. The maximum value of the equivalent stress in the slot fillet exceeded the yield strength of the titanium alloy BT3-1 at the temperature considered.

Our numerical results showed that the effects of the corner crack front position and the mode mixity should be considered in order to obtain correct conclusions. Figure 3 shows that under the operating loading conditions at a crack inclination angle close to $45^{\circ}$, the distributions of the equivalent stresses (Fig. 3a) and plastic strains (Fig. 3b) were nonsymmetrical due to mode mixity, and the plastic zone was larger in the slot key relative to the free surface of the disk.

In order to compare the behavior of the governing parameter along the corner quarterelliptical crack front we used a dimensionless variable $R$ in a range from 0 to 1 , where $R=$ 0.0 indicates the exterior surface of the compressor disk and $R=1.0$ is the slot key's inner surface. Fig. 4 shows the maximum equivalent plastic strain distributions along the crack front in the compressor disk. The results correspond to the initial (front 1) and final (front 3 ) corner crack positions for the various temperatures. Due to the change in the aspect ratio from $a / c=0.48$ to 0.68 for the corner crack semi-axes, the strain maximum shifted from the free surface of the disk $(R=0)$ to the inner surface of the slot key's bottom $(R=1)$. 


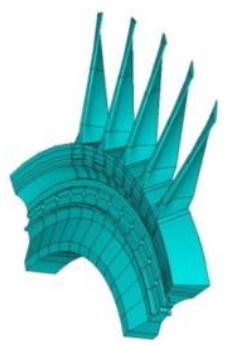

a)

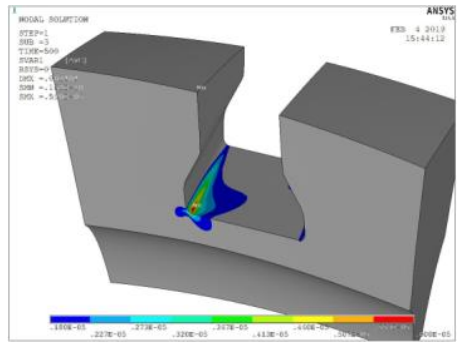

b)

Fig. 2. Global FE-model (a) and local (b) von Mises stress distributions in compressor disk section.

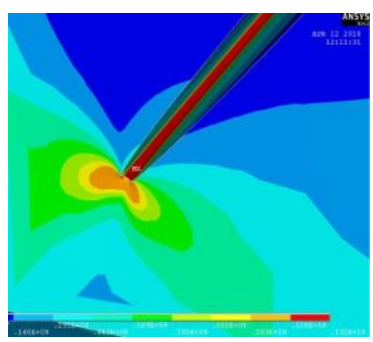

a)

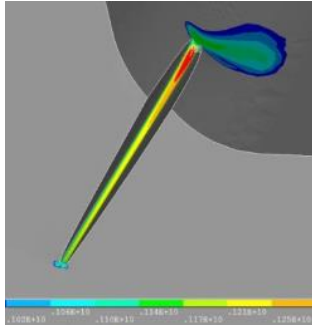

b)

Fig. 3. Mixed-mode distributions of equivalent stresses (a) and plastic strains (b) at the crack tip.

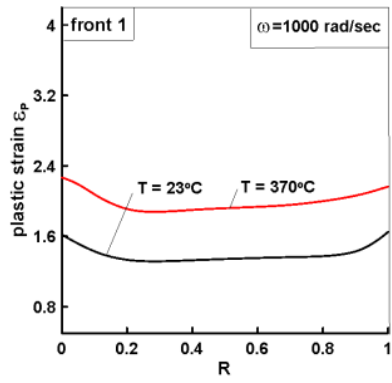

a)

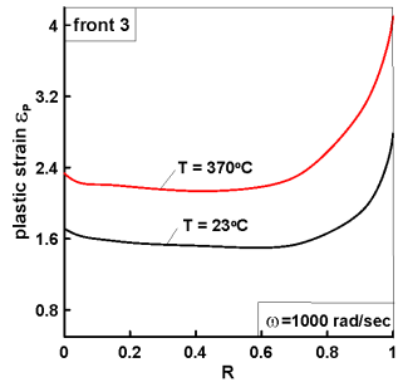

b)

Fig. 4. Maximum equivalent plastic strain distributions along the crack front.

The elastic equivalent mixed mode SIF $K_{\text {eqv }}$ along the corner crack was calculated using Eq. (7) for each of the surface flaw profiles and are shown in Fig. 5a. We found that the elastic SIFs changed by a moderate amount as a function of the crack front position and rotational speed. It should be noted that in the present study, the crack growth rate and fatigue lifetime were calculated as functions of room temperature $\left(23^{\circ} \mathrm{C}\right)$ and elevated temperatures $\left(300^{\circ} \mathrm{C}\right.$ and $\left.370^{\circ} \mathrm{C}\right)$. These calculations used the modulus of elasticity values at the corresponding temperatures. However, the behavior of the elastic SIF was virtually independent of the temperature.

The second parameter related to the fracture resistance of the compressor disk was the plastic SIF $K_{P}$, which was determined using Eqs. (7) and (8). In these calculations, in contrast to the elastic state, we considered the effects of the elastic-plastic properties of the titanium alloy BT3-1 according to Table 2 by employing the normalizing fatigue $I_{n}$-integral. Figure $5 \mathrm{~b}-\mathrm{c}$ shows the behavior of the dimensionless plastic SIF $K_{P}$ along the quarterelliptical corner crack fronts for the GTE compressor disk. Figure 6 shows the distributions 


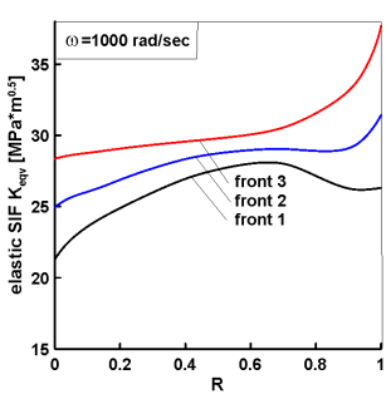

a)

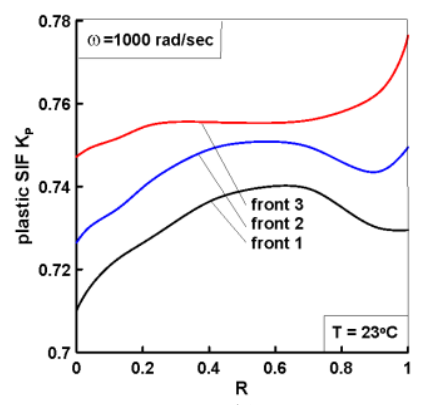

b)

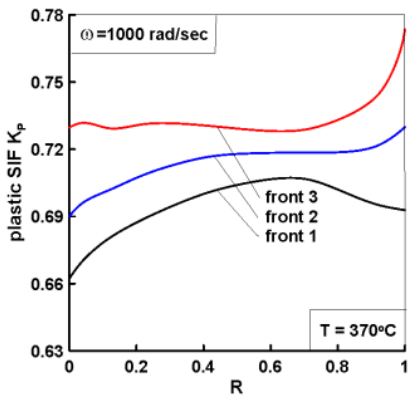

c)

Fig. 5. Elastic (a) and plastic (b-c) distributions of SIFs along the crack front.
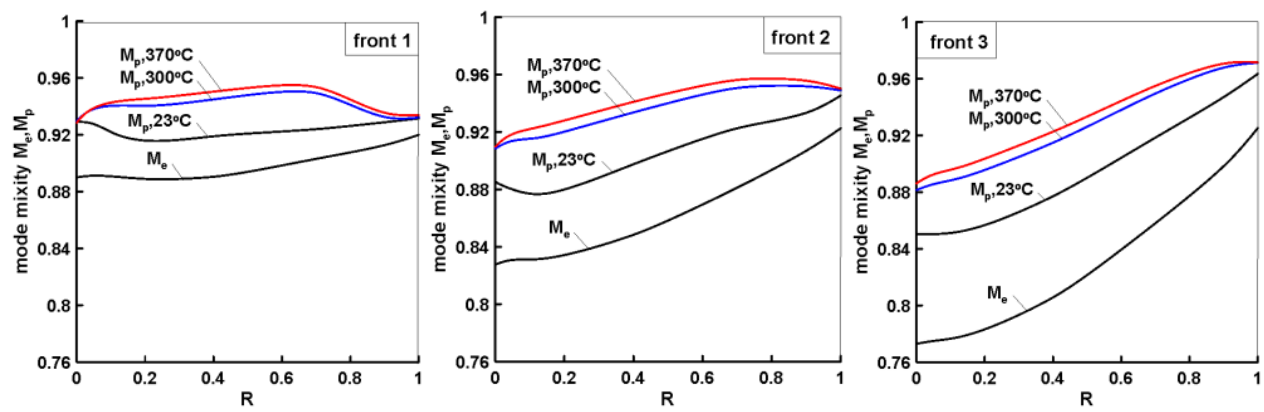

Fig. 6. Elastic and plastic mode mixity parameter distributions along crack front.

of elastic $M_{E}$ and plastic $M_{P}$ mode mixity parameters along three fronts in the compressor disk. Clearly, the plastic $M_{P}$ and the nonlinear SIF $K_{P}$ were sensitive to the main monotonic and cyclic plastic properties of the material as a function of the operating temperature of the compressor disk.

\section{Fatigue lifetime prediction}

Figure 7 compares the predicted change in the crack length $a$ on the free surface of the compressor disk (Fig. 7a) and the crack size $c$ along the slot key (Fig. 7b) as a function of the fatigue load cycles for the elastic and elastic-plastic solutions. The solid lines in Fig. 7 represent the predictions obtained based on the nonlinear SIF $K_{P}$, whereas the dashed lines represent the elastic solutions. The different combinations of the rotational speed, crack sizes and operation temperature listed in Table 1 were employed in this comparison.

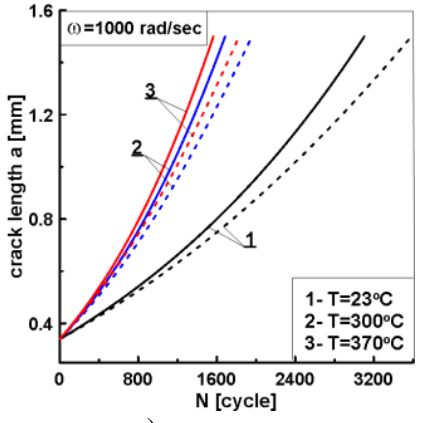

a)

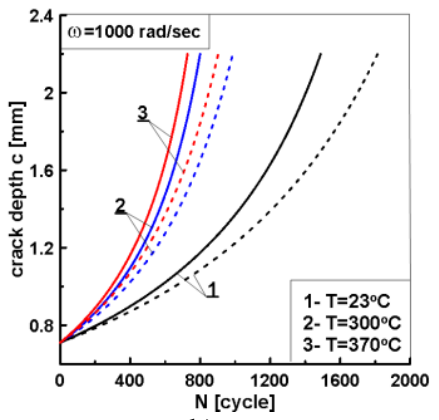

b)

Fig. 7. Fatigue lifetimes on the free surface (a) and slot surface (b) of the compressor disk. 
The maximum differences in durability for the elastic and elastic-plastic solutions corresponded to point $C$ at the corner front of the crack on the surface of the key slot. In all the cases considered with different rotation speeds and temperatures, the elastic solution obtained using the elastic SIF overestimated the lifetime compared with the numerical results based on the plastic SIF. The differences between the elastic and plastic solutions were $21.8-22.4 \%$ at room temperature and $23.7-24.0 \%$ at elevated temperatures. Predicting the crack growth rate of the aviation GTE compressor disk according to the nonlinear

fracture mechanics approach was much faster and the residual fatigue lifetime was lower compared with elastic modeling, thereby demonstrating that the low cycle fatigue material properties significantly affected the damage accumulation and growth in the fracture process zone ahead of the crack tip. Thus, the residual fatigue lifetime should be determined based on the nonlinear fracture mechanics criteria and parameters in order to analyze quarter-elliptical cracks at the corners an aviation GTE compressor disk under the operating loading conditions in order to estimate the crack growth rate.

\section{Conclusions}

In the present study, was assessed the residual lifetime for a titanium aviation GTE compressor disk with initial corner flaws based on the elastic-plastic crack tip singularity fields and full-size 3D finite element analyses to obtain fatigue crack growth predictions. We compared the assessments of the structural integrity of the rotating disk with the elastic and elastic-plastic solutions. Different combinations of the rotational speed, operating temperature, surface flaw form and size, and elastic-plastic properties of the titanium alloy BT3-1 were tested and compared. It is found that the traditional elastic crack growth models overestimated the residual fatigue lifetime compared with the nonlinear fracture mechanics approach, where the difference could exceed $24 \%$. The proposed method for assessing the lifetime of GTE disks based on the plastic SIF as a fracture resistance parameter has considerable practical importance.

\section{References}

1. V.N. Shlyannikov, A.V. Tumanov. Characterization of crack tip stress fields in test specimens using mode mixity parameters. Int J Fract, 185: 49-76 (2014)

2. V.N. Shlyannikov, N.V. Boychenko, A.V. Tumanov, A. Fernandez-Canteli. The elastic and plastic constraint parameters for three-dimensional problems. Eng Fract Mech, 12: 83-96 (2014)

3. F. Ellyin. Crack growth rate under cyclic loading and effect of different singularity fields. Eng Fract Mech, 25: 463-473 (1986)

4. K. Golos, F. Ellyin. A total strain energy density theory for cumulative fatigue damage. J Eng Mater Tech, 110: 36-41 (1988)

5. $n$ Code DesignLife 9 User Guide, HBM United Kingdom Limited (2013)

6. V.N. Shlyannikov, Elastic-Plastic Mixed-Mode Fracture Criteria and Parameters, Springer Verlag, Berlin (2003)

7. V.N. Shlyannikov, A.P. Zakharov, R.R. Yarullin. Structural integrity assessment of turbine disk on a plastic stress intensity factor basis. Int J Fatigue, 92: 234-245 (2016)

8. ANSYS Mechanical APDL Theory Reference Release 14.5, ANSYS, Inc. Southpointe, 275 Technology Drive, CanonBurg, PA (2012) 